\title{
HEMOENCEFALOGRAFIA CEREBRAL (HEG): FUNDAMENTOS TÉCNICOS APLICADOS AO JOGO LIFE
}

\section{ARTIGO ORIGINAL}

OLIVEIRA, Nathalia Alves de ${ }^{1}$

SILVA, Cintia Ramalho Caetano da ${ }^{2}$

OLIVEIRA, Nathalia Alves de. SILVA, Cintia Ramalho Caetano da. Hemoencefalografia Cerebral (HEG): Fundamentos Técnicos Aplicados ao Jogo Life. Revista Científica Multidisciplinar Núcleo do Conhecimento. Ano 05, Ed. 03, Vol. 09, pp. 31-50. Março de 2020. ISSN: 2448-0959, Link de acesso: https://www.nucleodoconhecimento.com.br/saude/hemoencefalografiacerebral

\section{RESUMO}

O Neurofeedback, uma técnica de treinamento cerebral, não invasiva, tem como objetivo a autorregulação neurofisiológica. O HEG é uma modalidade mais recente do Neurofeedback, criado em 1995, tem como foco o incremento intencional da oxigenação e perfusão sanguínea. A presente revisão tem como objetivo apresentar a técnica de Neurofeedback Hemoencefalográfico utilizado com o JOGO LIFE e suas possibilidades de aplicação no treinamento autorregulatório do lobo pré-frontal.

Palavras Chaves: Hemoencefalografia Cerebral, neurofeedback, Jogo Life.

${ }^{1}$ Graduada em Pedagogia pela Fetac. Especialista em Neuropsicopedagogia pela Universidade Cândido Mendes. Mestrado Gerontologia pela Funiber. Doutoranda em Psicologia pela lesla.

2 Mestre em Computação, Pós Graduada em Neuropsicopedagogia Clínica e Graduada em Pedagogia. 


\section{INTRODUÇÃO}

A técnica de neurofeedback HEG tem trazido grandes contribuições para a área de saúde, neurologia, fisiologia, psicologia e outras áreas. O neurofeedback é um procedimento/treinamento não invasivo e não medicamentoso, realizado por eletrodos ligados a um computador e fixados no couro cabeludo do paciente. Os eletrodos realizam uma leitura da atividade elétrica cerebral (EEG) dos neurônios de áreas e estruturas cerebrais que se deseja treinar, ou seja, áreas comprometidas que se deseja modificar a atividade cerebral para alcançar a normalidade. Um sistema computadorizado, com parâmetros de treino estabelecido, monitora a atividade cerebral captada pelos eletrodos e conduz essas áreas a ondas de normalidade. Essa é a chamada natureza do condicionamento operante da atividade eletroencefalográfica, ou simplesmente Neurofeedback.

Compreende-se que o neurofeedback pode ser eficiente no tratamento de diversos distúrbios psicológicos e comportamentais incluindo convulsões, ansiedade, drogadicção, insônia, ineficiências cognitivas, traumas, desordens de humor, transtorno obsessivo-compulsivo (TOC), desordens de personalidade, traumatismos cerebrais, dor crônica, artrite, epilepsia, dores de cabeça e enxaqueca, TDAH, e demais distúrbio neurológicas e cerebrais

$\mathrm{Na}$ atualidade, as modalidades de neurofeedback disponíveis para a clínica são a eletroencefalografia (EEG) e a Hemoencefalografía (HEG), onde o EEG se baseia em atividades de monitoramento e retroalimentação da atividade elétrica cerebral e HEG se baseia, conforme estuados atuais na técnica de monitoramento e retroalimentação do fluxo sanguíneo cerebral a qual colabora para o controle voluntario da oxigenação em módulos cerebrais especificamente escolhidos.

Tendo como ponto alvo o córtex pré-frontal, o equipamento de HEG é composto de uma faixa fixada através de um velcro, onde sensores realizam a leitura do fluxo sanguíneo cerebral, onde a faixa se posiciona nas regiões orbitais da testa, destacase a região giro orbito frontal, córtex pré-frontal ventromedial e ventrolateral (Fp1, Fp2, Fpz, F7). 
O objetivo da técnica é desenvolver no paciente a habilidade de controlar voluntariamente o fluxo sanguíneo na área em treinamento na finalidade de ativar uma área do cérebro, especificamente que consome mais oxigênio o que permitirá a ativação voluntária do exercício cerebral, incrementando a sinaptogênese e a angiogênese na região estimulada.

Destaca-se a grande facilidade na operação dessa técnica, sendo assim o neurofeedback HEG é indicado como a solução ideal para treinar o córtex pré-frontal. Sendo assim, o presente artigo tem como objetivo discorrer sobre a técnica de neurofeedback hemoencefalográfico (HEG neurofeedback), assim como traçar a história do HEG, os tipos de HEG, suas aplicações, e finalmente seus fundamentos técnicos aplicados ao jogo Life.

\section{ENTENDENDO O HEG}

O HEG (Hemoencefalografia) é uma técnica de neurofeedback relativamente nova. Inicialmente temos como base o neurofeedback, uma técnica específica de biofeedback, que surgiu em 1963, quando o professor Joseph Kamiya, da Universidade de Chicago, desenvolveu estudos treinando um voluntário com objetivo de reconhecer as ondas cerebrais alfa, e posteriormente alterar essas ondas. (SIEVER, 2008)

Os estudos de Joseph Kamiya permitiram fundar a área de treinamento de ondas cerebrais, que ficou conhecido como condicionamento operante da atividade eletroencefalográfica (EEG), ou somente neurofeedback. Kamiya é considerado o pai do neurofeedback. Já em 1971, Barry Sterman após a realização de testes em animais, treinou um epiléptico para controlar suas convulsões através do treinamento de um ritmo que foi nomeado sensório motor. (STOKES, 2010)

Na mesma época, Hershel Toomim, realizava estudos de medição de ondas cerebrais com mecanismos de biofeedback, em seu laboratório recém fundado, o Biofeedback Laboratories e o Biocomp Research Institute. Em 1994, Hershel Toomim, acidentalmente descobriu o controle consciente do fluxo sanguíneo cerebral. Dessa 
maneira, ele desenvolveu um dispositivo para aferir essas medidas, que chamou de Sistema de Hemoencefalografia de Espectrofotometria de Infravermelho Próximo, que somente em 1997 foi cunhado de Hemoencefalografia. (SIEVER, 2008)

O HEG pode ser realizado em duas modalidades, o HEG de espectroscopia por infravermelho próximo (NIR - Near InfraRed) e o HEG por infravermelho passivo (PIR - Passive InfraRed). O NIR foi desenvolvido pelo Dr. Hershel Toomim e mede as mudanças no nível local de oxigenação do sangue, ou seja, uma luz infravermelha penetra na camada superficial do cérebro, através da pele e crânio para avaliar a cor do tecido cerebral. Essa luz possui comprimentos de onda de 650 a 1000nm e mede as mudanças no nível local de oxigenação do sangue. (LONDERO; GOMES, 2014)

De acordo com Carvalho (2016, p.11) " HEG NIR o quão vermelho está o sangue circulante na região pela emissão de um fecho de luz infravermelha que penetra no crânio e reflete retornando ao equipamento". Assim nessa percepção o principal nutriente monitorado pelo NIR é o oxigênio. Isso porque, a luz capturada é medida e apresenta os níveis de saturação de oxigênio na região.

A partir dessas informações obtidas, elas são enviadas para um computador, e as sessões de neurofeedback possibilitam a manipulação consciente desse sinal. $O$ computador através de um sistema que realiza e expressa as leituras cerebrais realizadas apresenta em vermelho o sangue arterial oxigenado, e em azul o sangue venoso desoxigenado. A partir das informações obtidas sobre a cor e a temperatura do tecido pode-se entender a excelência do metodo de neurofeedback para treinamento da área pré-frontal. (LONDERO; GOMES, 2014)

Os equipamentos de neurofeedback HEG utilizados para o treinamento são de certa maneira simples. Não exige uma preparação previa da pele ou um produto adicional (como aplicação do gel), basta apenas que os eletrodos sejam colocados de maneira correta sob a região que será treinada, no caso a testa do paciente. As áreas prioritariamente treinadas com HEG neurofeedback são os sítios pré-frontais (Fp1, Fpz e Fp2) e frontais (F7 e F8, ver Lobos frontais). Na figura 1 destaca-se as áreas de treinamento do HEG, que são cinco pontos na região da testa. 
Figura 1 - Áreas treinadas com HEG - Pré-frontais (FP1, FPz e FP2) e Frontais (F7 e 8)

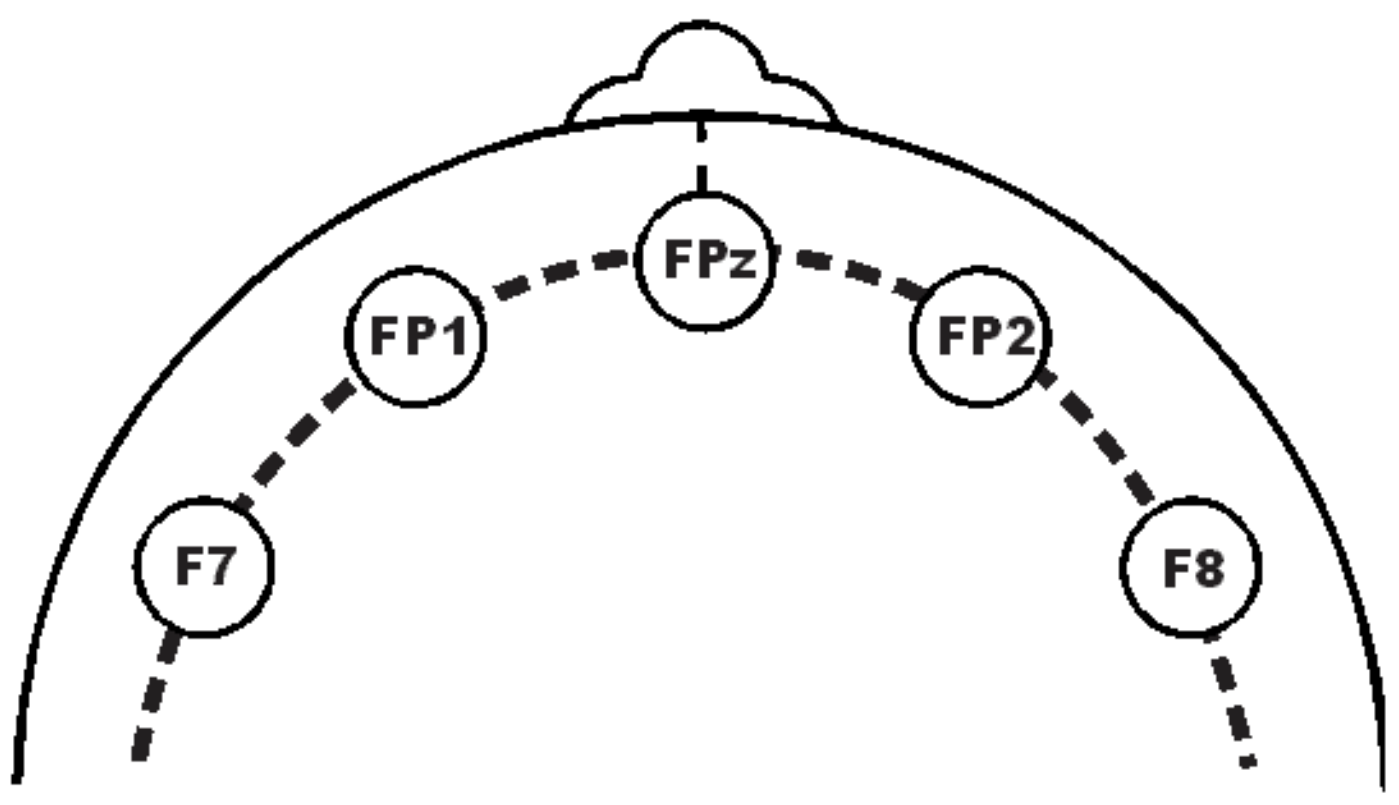

Fonte: (CARMEN, 2004)

O HEG por infravermelho passivo, também conhecido por PIR foi desenvolvido pelo Dr. Jeffrey Carmen, de Nova York. O HEG PIR é uma junção das técnicas do NIR aliadas a uma técnica conhecida como termoscopia, onde detecta-se a luz de uma faixa estreita do espectro infravermelho que corresponde à quantidade de calor que está sendo gerada por uma região cerebral ativa, bem como ao nível local de oxigenação do sangue.

O calor detectado pelo HEG PIR é proporcional à quantidade de açúcar a ser queimado para manter o aumento da taxa metabólica necessária para abastecer a atividade neuronal elevada. O HEG PIR tem uma resolução pior do que o HEG NIR e esse tratamento normalmente se concentra em aumentos mais globais no fluxo sanguíneo cerebral. (CARMEN, 2004) 
É importante entender como aumentar um determinado indicador. A resposta mais simples seria disser ao nosso paciente: "Faça subir esse indicador" e após alguns minutos haverá um aumento significativo. Toomim (2002) afirma que a resposta mais apropriada para esse aumento seria que tudo o que realmente queremos fazer, fazemos, sendo muito interessante o fato de que o nosso paciente baseado no desejo e na atenção concentrada consegue promover mudanças a nível cerebral. Isso porque HEG não está ligado a relaxamento, mas a atenção concentrada. A figura 2 mostra um paciente com o HEG em utilização.

Figura 2 - Paciente em sessão utilizando o HEG NIR

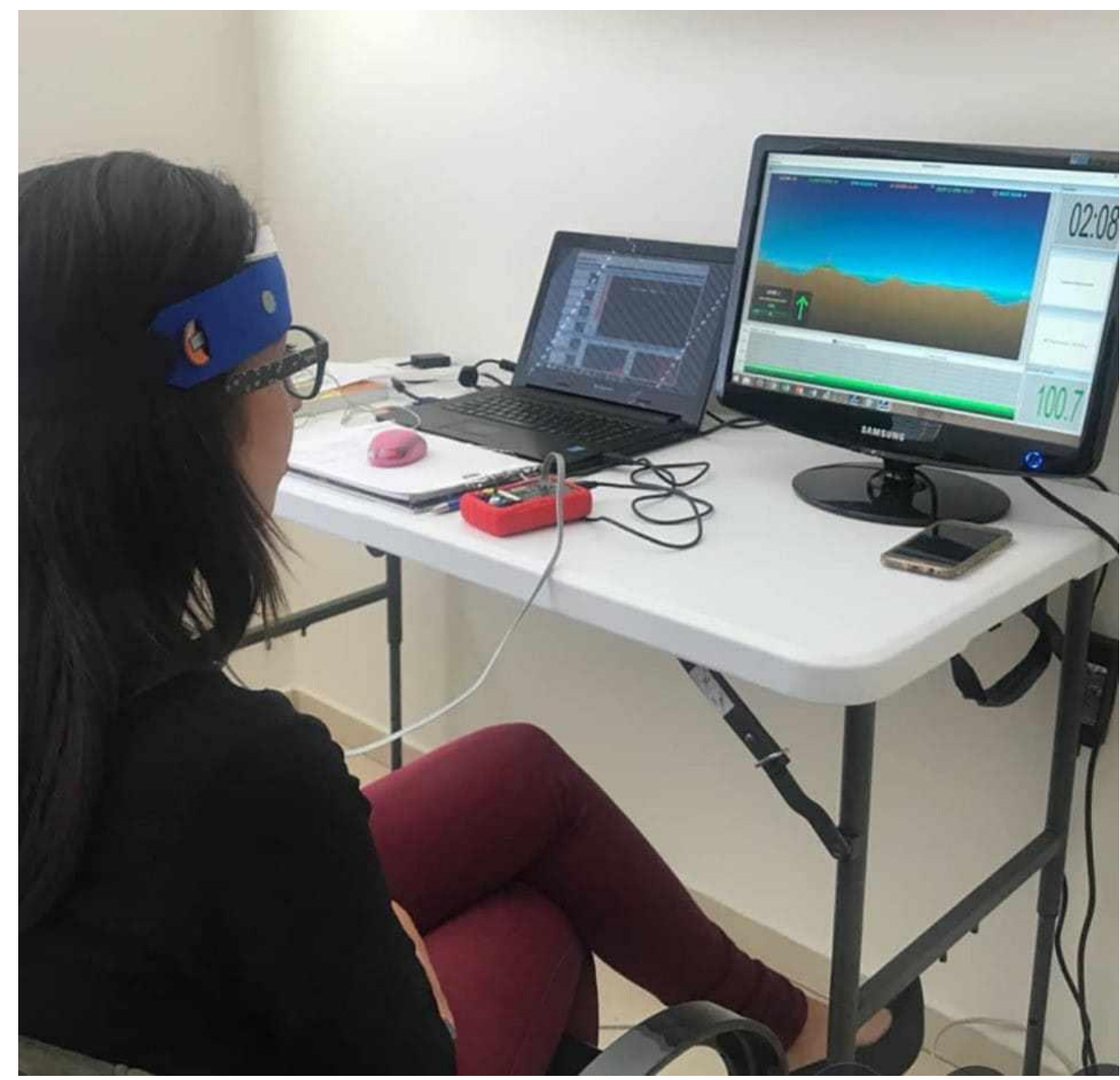

Fonte: (LONDERO; GOMES, 2014) 
Grande é a importância dos lobos frontais (região mais moderna do cérebro humano) nas funções executivas. "Atualmente é sabido que os módulos corticais responsáveis pelas funções executivas se localizam nos lobos frontais direito e esquerdo" (JUNIOR; MELO, 2011, p.44).

Sendo assim, a função dos lobos frontais é chamada de função executiva. O córtex pré-frontal é o responsável pela avaliação do sucesso ou fracasso das ações dirigidas a objetivos estabelecidos (GOLDBERG, 2002).

Entende-se que o mesmo está relacionado a execução da tarefa, na tomada de decisão, no sequenciamento, na memória de trabalho e na atenção, coordenando e integrando fatos aritméticos, recuperando dados da memória, de maneira a chegar à resposta esperada. $O$ córtex pré-frontal também é o grande amestro responsável pelo planejamento consciente das atividades motoras. Ele faz o controle motor de todos os nossos movimentos. (BRAIN-TRAINER, 2019)

Segundo Londero e Gomes (2014, p.45)

O incremento voluntário da perfusão sanguínea proporcionada pelo treinamento com HEG neurofeedback (tipo NIR) nas áreas pré-frontais tem como objetivo "forçar" o sistema de irrigação cerebral a ampliar a quantidade de vasos capilares nessa região, de forma a aperfeiçoar o funcionamento metabólico e, por consequência, melhorar suas funções, segundo o ponto de vista cognitivo.

De maneira mais ampla, o HEG tem como objetivo de treinar pacientes que apresentem déficits de atenção, dificuldades nas funções executivas e problemas na memória operacional. 


\section{FUNDAMENTOS TÉCNICOS}

\subsection{CONFIGURAÇÕES INICIAIS}

Inicialmente, um sistema de treinamento de neurofeedback HEG é composto de um computador e um módulo amplificador. $\mathrm{O}$ amplificador em linhas gerais realiza a captação dos sinais elétricos através dos eletrodos (escalpos) ligados por cabos a faixa na testa do paciente. $O$ amplificador transforma os sinais elétricos em sinais digitais, que posteriormente serão interpretados por um software específico e proporciona o feedback para o paciente.

Os amplificadores de Neurofeedback são classificados segundo a quantidade de canais que possuem, podendo ser de 2, 4 ou mais canais, ou apenas possuir a função HEG. Para o HEG o amplificador deve possuir a entrada para a conexão da faixa do HEG. As marcas mais utilizadas são: U-wiz, Pendant, Pocket, Peanut, Q-wiz (figura 3), Optima+, etc. Destacamos três tipos de amplificadores:

- Q-wiz de 4 canais, NIR ou PIR HEG, que atualmente é o dispositivo mais completo no mercado;

- X-wiz de 4 canais que permite ser utilizado com a faixa HEG e software para treinamento de HEG. Dois canais resistentes NIR HEG (Toomim) e um canal PIR (Carmen) mais Oximetria de Pulso. NIR e PIR podem ser simultâneos. 
Figura 3 - Amplificador Q-wiz

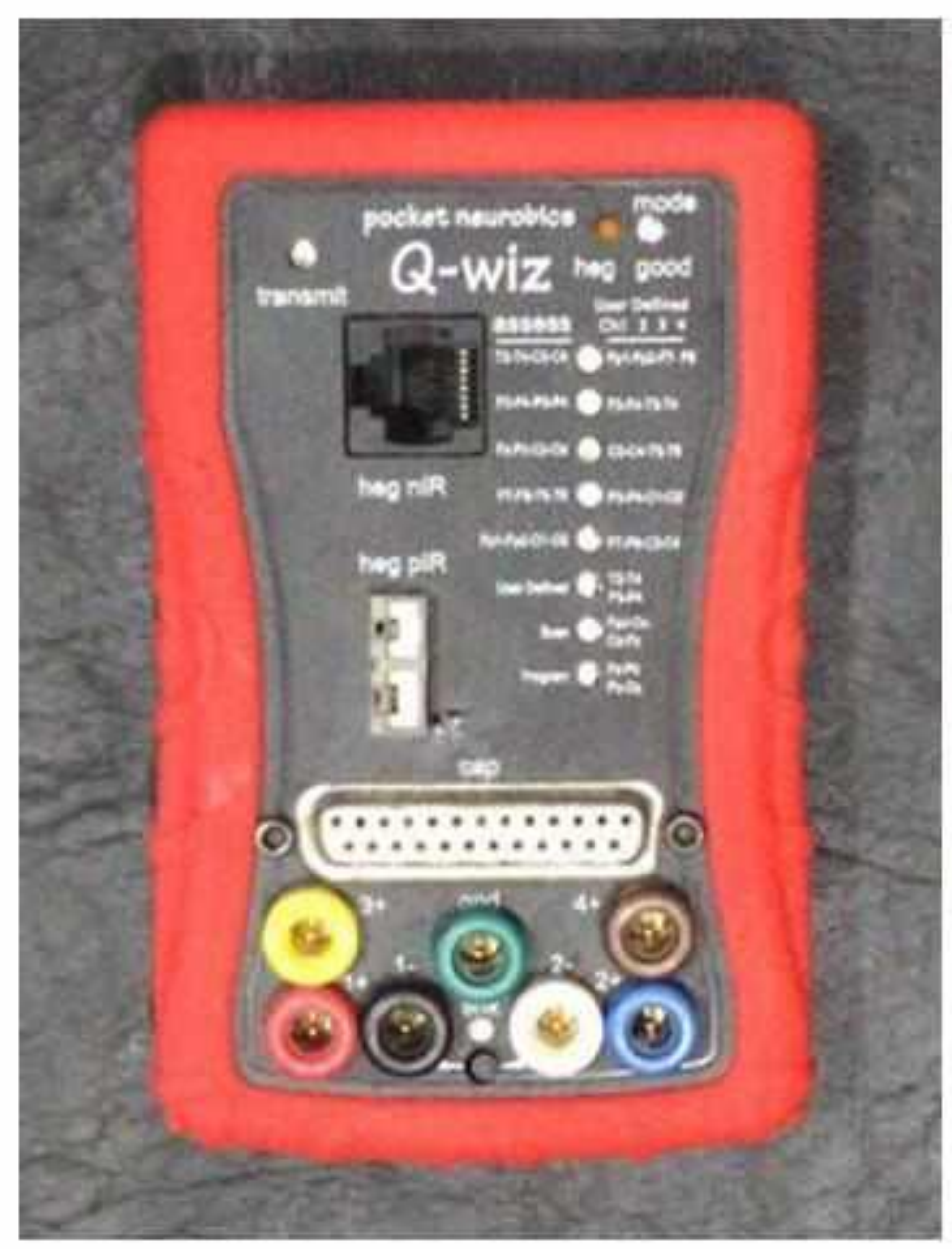

Fonte: (BRAIN-TRAINER, 2019)

O amplificador será conectado a faixa HEG, que será usada para aumentar a perfusão sanguínea nas áreas pré-frontais do cérebro com NIR. Isso pode ser feito utilizando um amplificador um Q-wiz, X-wiz, Peanut ou Pendant, o treinamento usa a mesma faixa de cabeça NIR HEG, conforme imagem abaixo. (BRAIN-TRAINER, 2019) 
Figura 4 - Faixa de HEG

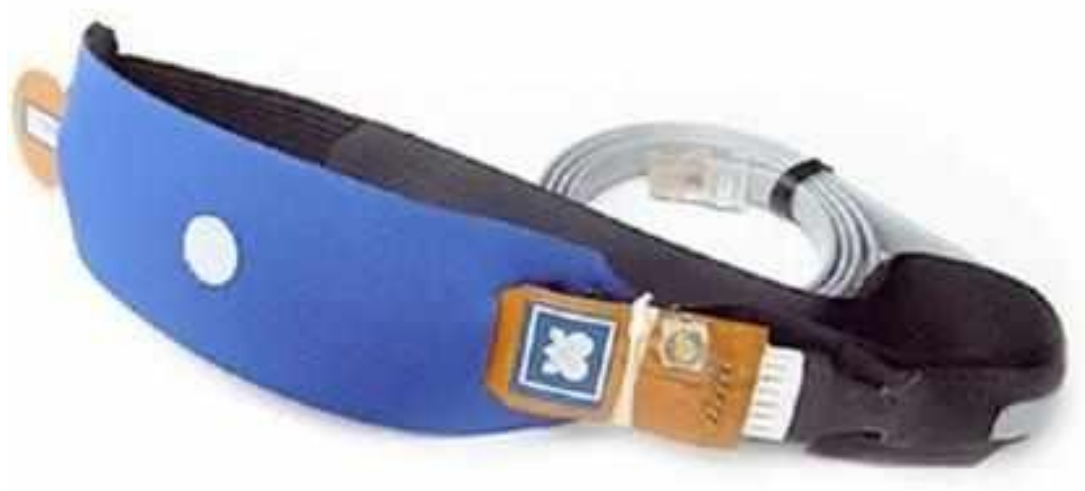

Fonte: (BRAIN-TRAINER, 2019, on-line)

O HEG é projetado para aumentar a oxigenação, ou seja, capacidade metabólica sanguínea das áreas treinadas com infravermelho próximo. Quando posicionamos a faixa na localidade a ser treinada, fazemos a seleção da área treinada para que a interface use o limiar da medida HEG para controlar aplicações (Flash, DVD, MPEG, Vídeo AVI, MP3, WAV e/ou som MIDI). O limiar é definido por uma captura manual após 70 segundos do usuário estar sem o uso do feedback. Quando iniciado o treinamento, se o usuário mantiver um nível de $10 \%$ ou mais acima da linha de base ou $2 \%$ ou mais abaixo da linha de base por 20 segundos, há uma redefinição automática da linha de base, para que o sistema possa desafiar o usuário durante todo o treinamento. (BIO-MEDICAL, 2019)

\subsection{INTERFACE BCI}

A interface $\mathrm{BCl}$ (Brain-Computer Interface) representa o processo de comunicação entre o cérebro humano e um computador, de maneira a interpretar a atividade em tempo real através de processamento de sinais e aprendizado de máquina. A partir desses dados processados é possível dar o feedback a partir da atividade cerebral on-line que pode ser usada para a auto-regulação do sujeito. 
As interfaces $\mathrm{BCl}$ são baseadas no controle bioelétrico, ou seja, são sistemas de gerenciamento de informações computacionais que medeiam a comunicação entre o cérebro e diferentes dispositivos. A tecnologia $\mathrm{BCI}$ permite que um humano controle um computador e outros dispositivos usando sinais cerebrais registrados na superfície da cabeça na forma de um eletroencefalograma (EEG) ou hemoencefalográfia (HEG), não havendo a transferência de dados pelos nervos e músculos. (KAPLAN et al., 2013)

Dando continuidade sobre as interfaces $\mathrm{BCl}$, os autores Kaplan et al., (2013, p.55) descrevem que:

As interfaces $\mathrm{BCl}$ são um sistema de gerenciamento, que registra os sinais cerebrais, analisa e os transforma em comandos que chegam aos dispositivos de saída para executar as ações desejadas. De acordo com a definição, a $\mathrm{BCl}$ é um sistema que mede a atividade cerebral e a transforma em um sinal de saída artificial, que substitui, recupera, aciona, dá suporte, informa ou melhora um sinal de saída natural e, dessa forma, altera as interações atuais de o cérebro com ambientes externos e internos.

O principal objetivo da tecnologia $\mathrm{BCl}$ está na substituição ou recuperação de funções úteis para indivíduos incapazes de realizá-las devido a distúrbios neuromusculares, como esclerose amiotrófica, paralisia cerebral, acidente vascular cerebral ou lesão medular. Além disso, permite estabelecer a comunicação entre áreas intactas do cérebro humano, o que permite compensar as funções motoras e sensoriais.

Existem muitos softwares $\mathrm{BCl}$ disponíveis no mercado, dentre eles destaca-se o Neuroserver, BioEra, BrainBay, BWView, Electric Guru, etc. Alguns desses são opensource (software abertos sem custo) e outros closed-source (software fechado com custo e suporte). (SIEVER,2008)

Assim conforme Goldberg (2002) um dos softwares BCI mais difundidos no Brasil é o BioExplorer, um software comercial criado pela CyberEvolution utilizado para o 
treinamento em biofeedback. O software é habilitado no computador via pen-drive com uma chave de segurança, onde através de arquivos de instalação permite a captação dos sinais fisiológicos. O ponto positivo que permitiu sua popularização pelo mundo é a facilidade na adaptação com vários tipos de equipamentos de hardware.

Figura 5 - Tela do BioExplorer

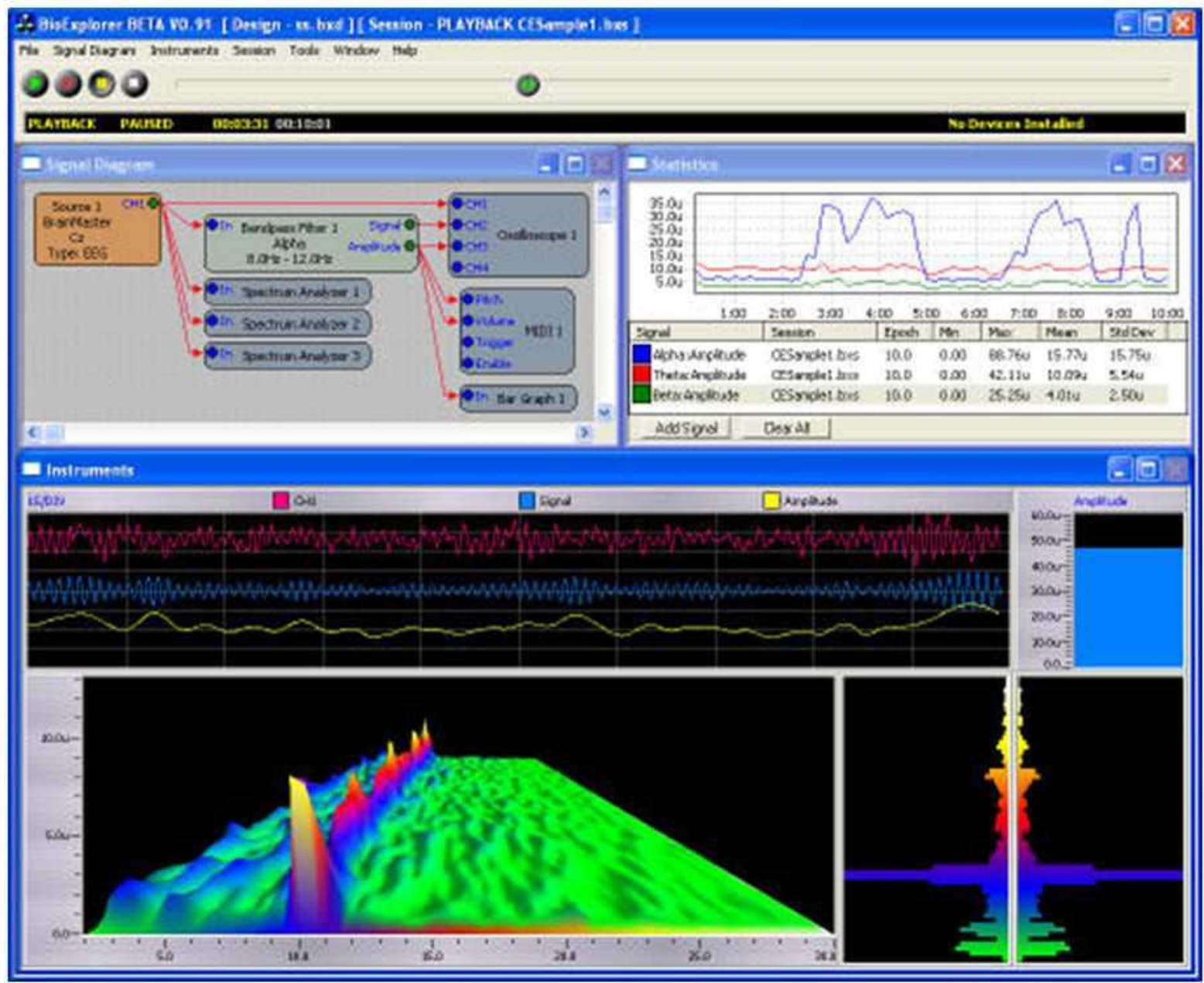

Fonte: (CYBER EVOLUTION, 2019, on-line)

\subsection{DESIGN}

O BioExplorer usa um 'Design' para informar o que fazer com os dados do seu dispositivo. O design é um protocolo de treino (interface), ou seja, cada design trabalha uma área a ser treinada. O design é comprado separadamente do software BioExplorer. O Design de Jogo mais usado para HEG utilizado no BioExplorer é o

Disponível em: https://www.nucleodoconhecimento.com.br/saude/hemoencefalografia-cerebral 
HEG Life. Ele é um design único exclusivo da Brain-trainer. Foi criado pelo Peter Van Deusen. (RIBAS, 2016)

Van Deusen, em 1994, desenvolveu uma técnica de treinamento cerebral baseada em um protocolo investigativo, chamado Curva de Aprendizagem (TLC) em neurofeedback. O modelo só se consolidou em 2001 onde o protocolo TLC foi criado a partir da leitura, interpretação e reprodução dos resultados de vários autores. (GOLDBERG 2002)

Van Deusen combinou seis categorias, no protocolo a ser usado por todos os treinadores que empregam o método TLC. São elas: 1) desconectados; 2) lobos temporais quentes; 3 ) inversões de alpha e beta; 4) bloqueado; 5) trancado; 6) filtrado ou 7) processamento. (SIEVER,2008)

Segundo Ribas (2016) a TLC mostra as relações entre as atividades elétricas, mentais e comportamentais nos pacientes e também fornece uma identificação detalhada dos padrões que podem ajudar os médicos na escolha dos tratamentos. Deusen desenvolveu várias aplicações para melhorar as possibilidades terapêuticas com Neurofeedback, sendo o Jogo LIFE uma delas.

\subsection{JOGO LIFE}

Esta sessão do artigo discorrera sobre as funcionalidades técnicas do jogo LIFE, criado por Peter Van Deusen. Toda a sessão tem como base o Manual do jogo, disponibilizado na documentação

O LIFE é um jogo utilizado especificamente nos sistemas NIR HEG, utilizado nos amplificadores HEG Pendant, no Peanut e nos da família WIZ. O jogo LIFE foi criado para funcionar no BioExplorer. O jogo LIFE ensina a ativar e relaxar o PFC (córtex pré-frontal), de maneira a aumentar os níveis de oxigênio do sangue no centro executivo do cérebro. Sendo assim, é preciso saber como "relaxar" e "ativar" o PFC. (RIBAS, 2016) 
Assim como os designs da Brain-Trainer utilizados no BioExplorer o LIFE faz uso do Flash Player. O objetivo de LIFE é marcar pontos, tendo como recompensa o acréscimo de animais no campo de treinamento. Quanto mais alto se conseguir subir ou quanto mais fundo se conseguir mergulhar, maior é a pontuação e maior é o porte do animal que aparece na tela.

Em linhas gerais, a linha de base do sistema é média de 100. O sistema captura a linha de base do paciente. A partir daí, quando o paciente ultrapassa a linha de base média própria, ele aumenta os pontos de atenção média, e aumenta a perfusão sanguínea.

Normalmente, a maioria dos pacientes iniciam os treinamentos aprendendo a subir. Porém pessoas com dificuldades de concentração, motivação, autocontrole, organização ou com baixa energia/depressão, o treinamento para subir pode ser uma tarefa de maior dificuldade. A partir do momento que o paciente pegar a sensação de "foco em ponto único", e se mostrar capaz de produzir e sustentar boas subidas, ele estará apto a tentar sessões de mergulho.

O estado de Mergulhar é o contrário do estado de Subir. Em vez de "foco mental em ponto único", o paciente deverá desfocar os olhos, muda a atenção da mente para a respiração no peito/barriga. Este é um estado de "Foco Aberto". No começo, treinar com os olhos fechados pode ajudar. Aos poucos o paciente pode aprender a manter este estado abrindo lentamente os olhos. Pacientes com o pensamento obsessivo, comportamentos compulsivos, ficando travado, com ansiedade ou excesso de intelectualização podem achar que é mais difícil mergulhar.

Em suma, o estado de Subir está relacionado com o foco fechado, foco em ponto único. E o estado de Mergulhar está relacionado ao foco aberto, no estar presente.

Depois que o paciente adquirir a capacidade de aumentar ou diminuir a linha do jogo, deixe os 2 botões ligados para melhorar a capacidade de transitar entre os estados. O jogo vai começar sempre com Subir, mas depois de 1-2 minutos ele irá informar ao paciente que é hora de Mergulhar. Comece com 1-3 ciclos em cada local de 
treinamento em Subir e então acrescente o Mergulhar. Clientes com enxaquecas podem achar a oscilação entre os estados mais útil, uma vez que imita o que acontece em muitas enxaquecas.

Cada sessão vai treinar 2-3 locais (F7 ou FP1, FPz, FP2 ou F8), começando com 2-3 minutos em cada local e aumentando para até 10 minutos por site. As sessões começam em dias alternados, passando para uma sessão a cada 3-4 dias, até chegar a uma sessão por semana, conforme crescem os períodos de treinamento. Pode-se variar em qual ponto quer começar em cada sessão. O manual do jogo LIFE disponível na documentação do sistema trabalho um exemplo para aplicação do Subir e Mergulhar nas sessões de treinamento, conforme figura 6.

Flgura 6 - Tela do BioExplorer

\begin{tabular}{|r|l|l|}
\hline \multicolumn{3}{|c|}{ Exemplo } \\
\hline 0 & Apenas Subir & $3 \mathrm{~min}$ \\
\hline 0 & Apenas Mergulhar & $3 \mathrm{~min}$ \\
\hline 1 & Subir/Mergulhar & $3 \mathrm{~min} / 2 \mathrm{~min}$ \\
\hline 2 & Subir/Mergulhar & $3 \mathrm{~min} / 2 \mathrm{~min}$ \\
\hline 3 & Subir/Mergulhar & $3 \mathrm{~min} / 2 \mathrm{~min}$ \\
\hline 4 & Subir/Mergulhar & $3.5 \mathrm{~min} / 2 \mathrm{~min}$ \\
\hline 5 & Subir/Mergulhar & $3.5 \mathrm{~min} / 2 \mathrm{~min}$ \\
\hline 6 & Subir/Mergulhar & $3.5 \mathrm{~min} / 2 \mathrm{~min}$ \\
\hline 7 & Subir/Mergulhar & $4 \mathrm{~min} / 2 \mathrm{~min}$ \\
\hline 8 & Subir/Mergulhar & $4 \mathrm{~min} / 2 \mathrm{~min}$ \\
\hline$\ldots$ & assim por diante, não mais do que 10 \\
minutos por sítio & \\
\hline
\end{tabular}

Fonte: (Manual do Jogo LIFE)

$\mathrm{Na}$ figura 7 tem-se a tela de configurações para o treinamento do jogo LIFE. No item 1 temos os botões de configurações de treinamento (local, tempo de subida). No item 
2 temos a opção de salvar os dados para começar a ler os dados no BioExplorer. No item 3 temos a opção de conectar o jogo ao BioExplorer e iniciar o treinamento. No item 4 temos a opção de capturar Linha de Base e iniciar o treinamento. Após treinar um ponto, deve-se selecionar o botão "Stop" e começar a treinar o próximo local repetindo os mesmos passos.

Figura 7 - Tela de Configuração do Treinamento do LIFE

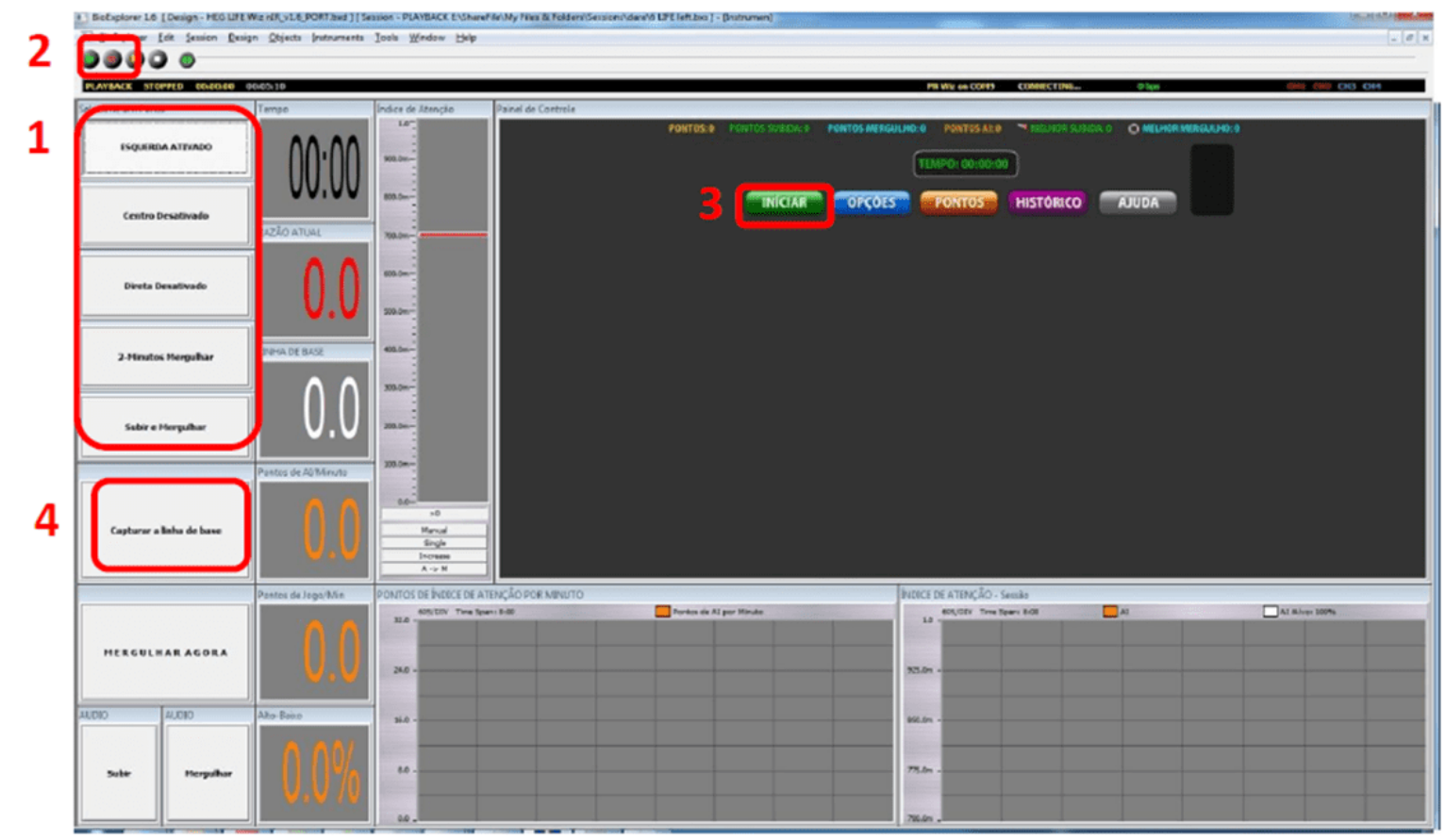

Fonte: (Manual do Jogo LIFE)

Ao final de cada rodada do jogo (após treinar vários minutos num ponto), o treinador deverá selecionar a opção "Parar BioExplorer", será exibido nas 2 janelas a pontuação para este jogo e para os anteriores, conforme figura 8. Ao clicar no botão "Histórico" no painel de controle, o treinador poderá rever a sessão. $O$ gráfico pode ser rodado ou pausado para apontar ocorrências específicas. Também é possível avançar ou retroceder e aumentar ou diminuir a escala clicando nas flechas para manter a área desejada na tela. 
Figura 8 - Tela de Pontuação do Jogo LIFE

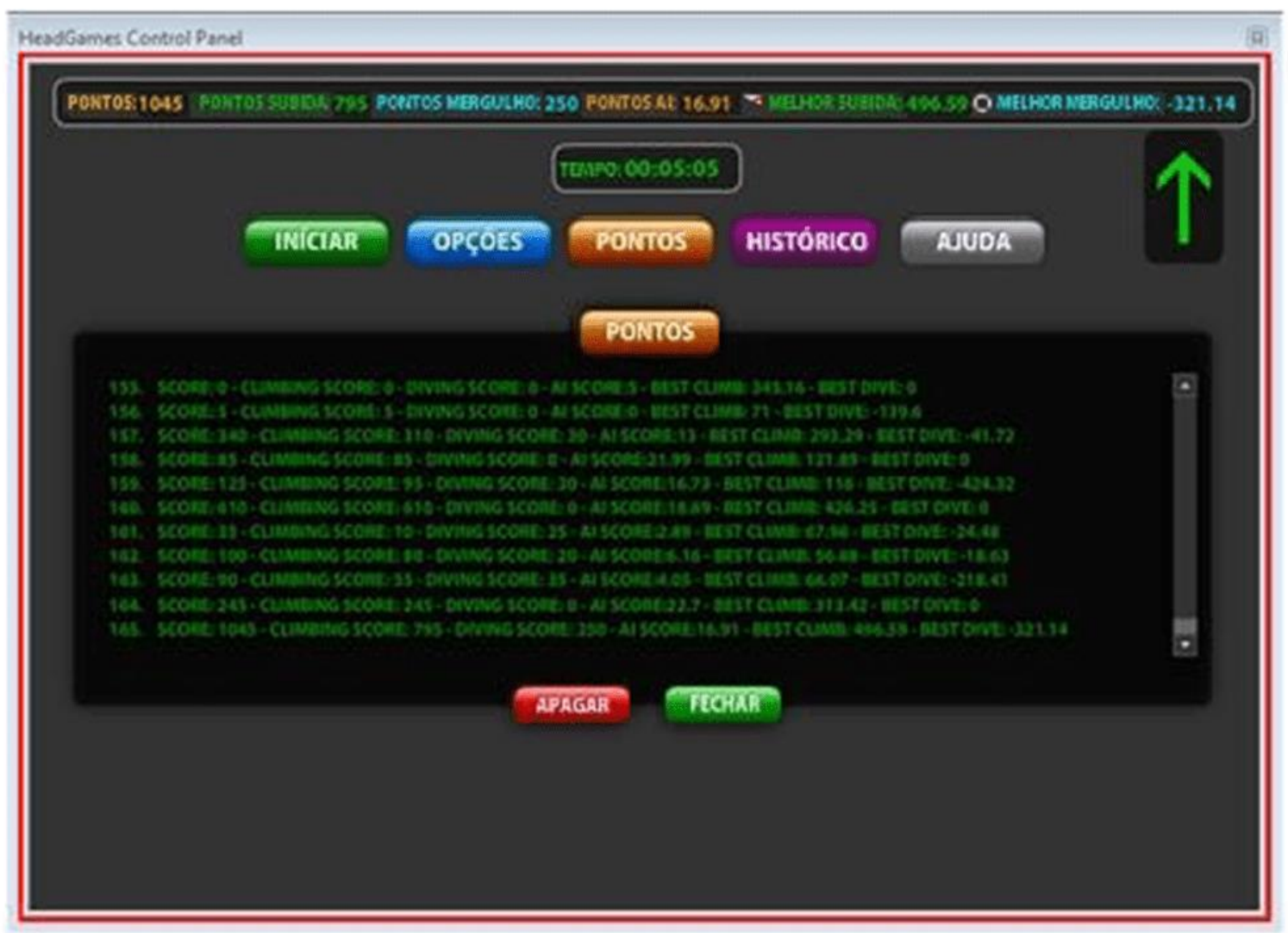

Fonte: Manual do Jogo LIFE

O jogo LIFE possui uma planilha chamada "auto-session-graphing", que carrega automaticamente os dados gravados para analisar os resultados do treinamento em várias sessões. A figura 9 apresenta os gráficos gerados com os resultados dos treinamentos do Jogo LIFE, relacionados a estabilidade, gama e controle. 
Figura 9 - Gráficos gerados com os resultados dos treinamentos do Jogo LIFE

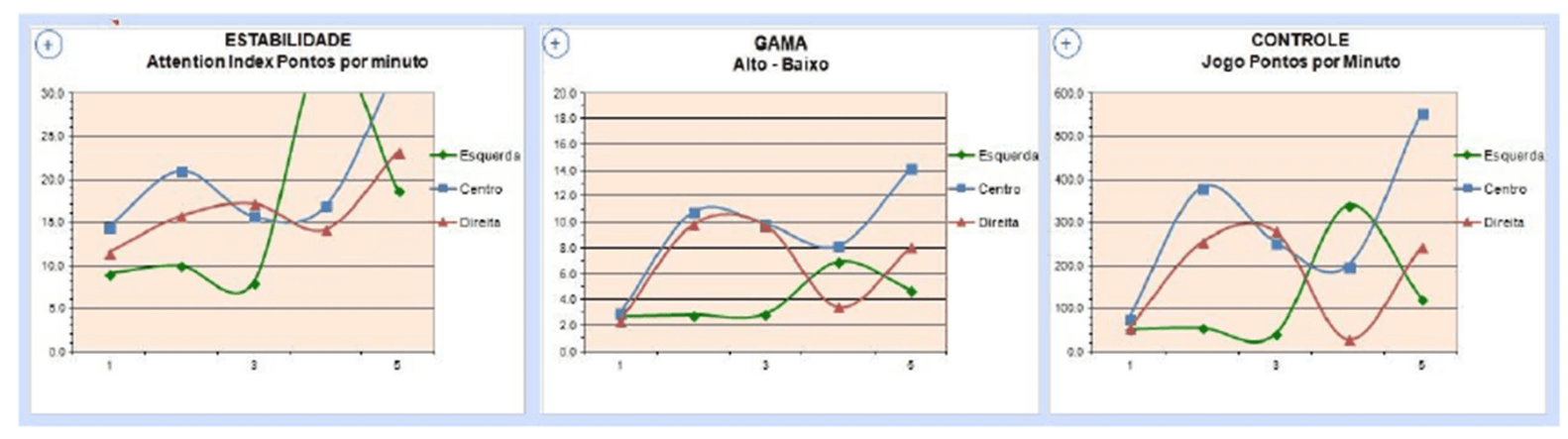

Fonte: (Manual do Jogo LIFE)

Em relação aos controles do treinador, tem-se várias opções disponíveis para que o treinador ajuste a melhor opção de configuração para o sucesso do treinamento. $O$ treinador inicialmente deve selecionar qual lado será treinado, selecionando dentre as opções de centro, direita ou esquerda. Outra configuração disponível é o Tempo de subida, no modo "rocking", o treinador que controla o tempo de subida.

A opção "Subir e Mergulhar/Apenas Mergulhar" deve ser utilizada para fazer apenas o mergulho. Quando o paciente conseguir controlar melhor a subida e descida comece um treinamento em "rocking" para melhorar sua capacidade de mudar tranquilamente de um estado para o outro. A opção "Mergulhar Agora" deve ser utilizada quando o paciente já tiver subido por todo o período desejado, ou quando apresentar sinais de cansaço, clique neste botão para iniciar um período de relaxamento.

O tempo de mergulho será de 1 ou 2 minutos de acordo com a escolha do treinador. Em seguida ele iniciará uma nova subida.

A opção "Painel de Controle" permite ao treinador que estiver usando um segundo monitor controlar o Flash Game. Botões irão permitir que o treinador inicie (Start) ou pare (Stop) o jogo. Training Options (Opções de Treinamento), mostra o score (placar) ou history (histórico) do jogo que acabou de acontecer. O botão Help (ajuda) serve para esclarecer dúvidas sobre o jogo. Options (opções), o treinador pode mudar a capacidade de resposta da tela dos clientes cujo sinal tem variações muito intensas 
ou muito pequenas a fim de torná-la visualmente mais útil. $O$ treinador também pode mudar o tempo de sessão e ajustar os alvos dos 5 níveis do jogo.

Durante o jogo vários instrumentos de exibição ficam disponíveis para dar informações ao treinador. O Timer da Sessão fica no canto superior esquerdo da tela do treinador. Ele exibe a duração da sessão de treino e é reiniciado quando o botão Capture Baseline (Capturar Linha de Base) é pressionado. Abaixo dele está o valor atual da razão de red/infrared e a linha de base. Quando a linha de base é capturada, o medidor LINHA DE BASE vai mostrar a relação no tempo como uma constante. Este é o valor que o paciente precisa ficar acima (ou abaixo para mergulho).

Mais três medidores em laranja mostram os três valores mais úteis para avaliar o progresso em todas as sessões: (1) Pontos de Índice de Atenção / Minuto Estabilidade; (2) Pontos de Jogo /Minuto - Controle; (3) Variação Máxima Alta/Baixa - Variação.

O Índice de Atenção (Al) é uma medida de quão consistentemente a razão HEG está aumentando. $100 \%$ é o melhor. O limiar Al fica à esquerda do painel de controle. Está fixado em $70 \%$ e não deve ser alterado. A barra fica laranja quando o Al do cliente fica acima de $70 \%$. É quando as pontuações do jogo ocorrem. Abaixo do painel de controle estão os gráficos de tendência, mostrando os pontos de Al por minuto e em toda a sessão.

A janela do jogo mostra um personagem com o desenho de um cérebro, sempre no canto direito da tela, correndo (quando estiver subindo) ou mergulhando (quando estiver descendo). O paciente pode acompanhar a evolução de seu treino comparando o lugar onde seu cérebro está com os lugares por onde ele passou anteriormente. Na terra aparecem coelhos, cachorros, leões, cavalos e elefantes que surgem cada vez que o cérebro apresentar desempenho igual ou melhor durante um segundo inteiro. Conforme o cliente sobe os animais mudam, valendo uma quantidade maior de pontos pra cada um. Na água aparecem peixes, tartarugas, tubarões, golfinhos e baleias. Cada vez que o cliente atinge novo recorde aparece na tela uma bandeira (se ele estiver subindo) ou uma boia salva-vidas (se ele estiver descendo). 
Uma seta no canto inferior esquerdo mostra se o treino é para subir ou descer. A janela Level mostra em qual dos 5 níveis o paciente está no momento, o alvo a ser atingido para mudar de nível e o progresso. Quando o alvo da pontuação é atingido um troféu aparece e o nível muda. A pontuação vai ficando mais difícil a cada nível e o paciente tem que sustentar seu desempenho por mais tempo para que um novo animal apareça.

No topo da tela o paciente pode acompanhar qual a sua pontuação geral, sua pontuação de subida (climbing score), de mergulho (diving score), sua maior pontuação de subida (best climb), de mergulho (best dive), e o número de Al pontos por minuto - uma medida de estabilidade. O Gráfico de Tendência na parte inferior da página mostra a relação entre o treinamento e a linha de base. Esta linha de tendência segue o chão na tela de jogo do LIFE.

O jogo LIFE vem com seus próprios sons para pontuar recorde de escalada e mergulho, mudança de direção e aumento de nível. O design LIFE faz um som suave (“ding') quando o Índice de Atenção é pontuado. Isso indicará que o desenho do cérebro está indo na direção certa, mesmo se ele estiver indo para o lado errado da linha de treinamento (pra água ao invés de escalar).

Na pasta de Documentos\Brain-Trainer é possível encontrar o LIFE CYCLE em .PDF e Excel para manter o controle de tempo de treinamento. É importante manter uma gravação para cada cliente em sua pasta de sessões. A meta é 300 minutos de treinamento de escalada. $O$ treinador deve registrar o número total de minutos de escalada para cada sessão. O LIFE CYCLE possui um guia para o cumprimento do treinamento. $\mathrm{O}$ arquivo Excel conta automaticamente os minutos da escalada e mantém um total atualizado.

Há três medidas primárias que rastreamos: Pontos Al por minuto (Estabilidade), Pontos de jogos por minuto (Controle) e Variação máxima de Alto para Baixo.

I. Estabilidade: pontos Al são uma medida da capacidade do seu cérebro de MANTER o estado desejado. A cada segundo, o LIFE gera o seu sinal de HEG 10 
vezes por segundo. Se cada medida é igual ou maior que a última (subindo) ou igual ou menor que a última (mergulhando) por um segundo, o gráfico Al na tela do treinador alcançará o topo - Al de 100. Então, se continuar por mais meio segundo sem ir na direção errada, você marca um ponto Al. O momento que o cérebro mostra uma mudança de direção - até mesmo 1 degrau - o Al cai, e você deve manter a subida ou o mergulho por um segundo novamente para conseguir começar a marcar pontos Al. Há dois gráficos na parte inferior da página do treinador. Você pode olhá-los depois que a sessão for finalizada para ver o quão continuamente foi capaz de manter o estado desejável.

II. Controle: Quanto mais estável a capacidade de seu cérebro de subir ou mergulhar, mais animais você colocará a cada minuto. Porém animais também têm valores de pontuação. Quando começa, você colocará (por exemplo) coelhos na tela -- cada um valendo 5 pontos. Se subir ou descer muito, indicando uma fraqueza no seu sistema de perfusão que abastece seus neurônios com sangue, você pode encontrar-se sempre colocando coelhos -- só coelhos. Mas à medida que sobe mais adiante, começa a colocar cachorros, depois leões, em seguida cavalos, e finalmente elefantes. Cada cachorro vale 10 pontos, leões 15, cavalos 20 e elefantes 25. Quanto mais alto (ou mais profundo) você for, mais Pontos do Jogo irá marcar para cada ponto Al. Então, Pontos do Jogo/Minuto é uma medida do quão fortemente você foi capaz de ativar ou desativar E quão consistentemente foi capaz de manter o estado. Ambos os pontos $\mathrm{Al}$ e os Pontos do Jogo são divididos pelo número de minutos de treinamento, então eles podem ser comparados em um segmento de minuto e um segmento de 10 minutos.

III. Variação: À medida que está subindo ou mergulhando, além do som "shing" quando marca um ponto, você também pode ouvir um "ding". Se está assistindo a tela, verá uma bandeira pequena (se estiver subindo) ou uma Bóia (se estiver mergulhando) aparecer na paisagem e rapidamente verá um valor numérico. Isso diz que você alcançou uma nova pontuação máxima ou mínima para aquela sessão. A terceira medida para o seu desempenho é o quão alto é capaz de subir quando está subindo, e o quão profundo é capaz de mergulhar quando está mergulhando. Estes 
são medidos contra a base de referência de subida e mergulho. $O$ ideal é que você seja capaz de alternar rapidamente para frente e para trás da concentração para a consciência (subindo e mergulhando) e que é capaz de manter cada estado quando está nele. A variação mede a porcentagem mais alta acima da linha de base que você alcançou quando subindo até a porcentagem mais profunda abaixo da linha de base quando mergulhando. Quanto maior for a variação, mais eficientemente o seu córtex pré-frontal será capaz de funcionar.

\section{CONCLUSÃO}

Os avanços tecnológicos e clínicos possibilitam que novos recursos venham surgindo tanto para reabilitação quanto para performance. O Neurofeedback emerge como uma destas possibilidades proporcionando a autorregulação neurofisiológica, mais recentemente o surgimento da modalidade sanguínea, o HEG trouxe mais possibilidades para a reabilitação e otimização das funções executivas.

Através das revisões literárias é possível ressaltar que os resultados de aplicações em outros países sejam bem significativos. Existe uma escassez de aplicações acadêmicas de cunho nacional. Há a necessidade de averiguações nacionais que apresentem avaliação, treinamento, e reavaliação, além de um grupo de controle. Sendo assim, se faz relevante a investigação científica para trazer um respaldo relacionado a sua eficiência no campo terapêutico.

\section{REFERÊNCIAS}

AGUIAR, S. Neurofeedback: Uma Opção não Medicamentosa para o Tratamento do TDAH. Revista de Villegagnon. 2014.

BRAIN-TRAINER. Disponível em <https://brain-trainer.com>. Acessado em 05/06/2019.

BIO-MEDICAL. Disponível em <https://bio-medical.com>. Acessado em 14/07/2019. 
CARMEN, J. (2004). Passive infrared hemoencephalography: four years and 100 migraines. Journal of Neurotherapy, 8 (3), 23-51.

CARVALHO. T. Utilização do Neurofeedback como Recurso Terapêutico não Farmacológico em Adolescentes e Adultos Jovens com Migrânea. Dissertação (Mestrado) - Universidade Federal do Pernambuco, CCS. Programa de Pósgraduação em Saúde da Criança e do Adoslescente. Recife, 2016. 95 f.

CYBER EVOLUTION. Disponível em <http://www.cyberevolution.com>. Acessado em 05/06/2019.

GOLDBERG, E. (2002). O cérebro executivo: lobos frontais e a mente civilizada. Rio de Janeiro: Imago.

JUNIOR, C.; MELO, L. Integração de Três Conceitos: Função Executiva, Memória de Trabalho e Aprendizado. Psicologia: Teoria e Pesquisa Jul-Set 2011, Vol. 27 n. 3, pp. 309-314.

KAPLAN, A.Ya.; KOCHETOVA, A. G.; SHISHKIN, S. L.; BASYUL, I. A.; GANIN, I. P.; VASILEV, A. N.; LIBURKINA, S. P. Experimental and theoretical foundations and practical implementation of technology brain-computer interface. Byulleten' sibirskoy meditsiny 2013; 12(2): 21-29.

LONDERO, I.; GOMES, J. S. Neurofeedback hemoencefalográfico (HEG): Possibilidades de Aplicações no Campo da Saúde. Ciências e Cognição, v. 19, n. 3, p. 307-314, 2014.

RIBAS, Valdenilson Ribeiro; RIBAS, Renata de Melo Guerra; MARTINS, Hugo André de Lima. The Learning Curve in neurofeedback of Peter Van Deusen: A review article.Dement. neuropsychol., São Paulo, v. 10, n. 2, p. 98-103, June 2016

Available from <http://www.scielo.br/scielo.php?script=sci_arttext\&pid=S1980$57642016000200098 \&$ Ing=en\&nrm=iso $>$. Access

on 08 June 2019. http://dx.doi.org/10.1590/S1980-5764-2016DN1002005. 
SIEVER, D. (2008). History of biofeedback and neurofeedback: the Hershel Toomim story. Biofeedback, 36 (2), 74-81.

STOKES, D. A; LAPPIN, M. S. Neurofeedback and biofeedback with 37 migraineurs: a clinical outcome study. Behavioral and brain functions : BBF, v. 6, p. 9, jan. 2010.

TOOMIM, H. (2002) Neurofeedback with hemoencephalpography (HEG) Explore! For the Professional, 11 (2), 19-21.

Enviado: Fevereiro, 2020.

Aprovado: Março, 2020. 